\title{
Further evidence for a large glue component in the $\mathbf{f}_{0}(\mathbf{1 5 0 0 )}$ meson
}

\author{
Claude Amsler ${ }^{1}$ \\ Physik-Institut der Universität Zürich, Winterthurerstrasse 190, \\ CH-8057 Zürich, Switzerland
}

\begin{abstract}
We argue that the experimental decay rates of the $f_{0}(1500)$ meson into two pseudoscalar mesons and into two photons are incompatible with a quark-antiquark state and that the $f_{0}(1710)$ meson is dominantly an $s \bar{s}$ state.
\end{abstract}

Key words: Glueball, meson, scalar, f0(1500); 12.38.Qk, 12.39.Mk,14.40.Cs

\section{Introduction}

Several $0^{++}$resonances were established recently, far too many to be accommodated in the ground state scalar nonet [1]. Below $1600 \mathrm{MeV}$, four isospin zero resonances are required to describe the isoscalar $\pi \pi \mathrm{S}$-wave: the very broad $(\sim 600$ $\mathrm{MeV}) f_{0}(600)$ (or $\left.\sigma\right)$, the narrow $f_{0}(980)$ at the $K \bar{K}$ threshold, the broad $(\sim 400$ $\mathrm{MeV}) f_{0}(1370)$, and the comparatively narrow $(109 \pm 7 \mathrm{MeV}) f_{0}(1500)$. The latter, first observed in $\bar{p} p$ annihilation at rest, was reported in many other reactions and is among the best established unstable mesons. For example, it is observed to decay into $\pi \pi$ [2,3], $K \bar{K}$ [4], $\eta \eta$ [5], $\eta \eta^{\prime}$ [6] and $4 \pi$ [7] in $\bar{p} p$ annihilation at rest, and in $p p$ central collisions at $450 \mathrm{GeV}$, into $\pi \pi$ and $K \bar{K}$ [8], $\eta \eta$ [10], $\eta \eta^{\prime}$ [9] and $4 \pi$ [11]. It also reported in an analysis of the $D_{s} \rightarrow 3 \pi$ Dalitz plot [12] and in the $4 \pi$ mass distribution in $J / \psi \rightarrow 4 \pi$ radiative decays [13]. It was, however, not observed so far in $\gamma \gamma$ collisions [14,15].

The small branching ratios for $K \bar{K}$ decay - compared to $2 \pi$ - of $f_{0}(1370)$ and $f_{0}(1500)$ point to both states being compatible with $u \bar{u}+d \bar{d}$ structures, while only one can be accommodated in a SU(3) nonet. In an earlier work [16] we suggested

$\overline{1}$ e-mail address: Claude.Amsler@ cern.ch

url: http://afuz01.cern.ch/zuerich.html 
that three isoscalar mesons in the $1500 \mathrm{MeV}$ mass region, a $u \bar{u}+d \bar{d}$ state, an $s \bar{s}$ and the ground state scalar glueball predicted by lattice gauge theories [17] mix to produce $f_{0}(1370), f_{0}(1500)$ and the at that time still to be identified third scalar state. In this scenario, a large $(\geq 50 \%)$ gluonic component was suggested in the $f_{0}(1500)$ wavefunction, while $f_{0}(1370)$ remained largely $u \bar{u}+d \bar{d}$, and the missing state mainly $s \bar{s}$. This was inferred (i) from the apparent absence of $K \bar{K}$ signal in earlier bubble chamber exposures suggesting a cancellation between amplitudes, (ii) from the narrow width of $f_{0}(1500)$ compared to the other known scalars, and (iii) from the observation of an isovector $a_{0}(1450)$ in this mass region. In this model the ground state $\left(1^{3} P_{0}\right)$ scalar nonet was therefore made of $a_{0}(1450), f_{0}(1370)$, an $s \bar{s}$ state around $1700 \mathrm{MeV}$ (both with small glue components), and $K_{0}^{*}(1430)$, while $f_{0}(1500)$ was mainly glue.

On the other hand, the $f_{0}(980)$ and $a_{0}(980)$ could be $K \bar{K}$ molecules or four-quark states [18], or together with $f_{0}(600)$, meson-meson resonances [19]. Alternative mixing schemes also imbedding quarkonia $f_{0}(1370)$ and $f_{0}(1500)$ have been proposed [20,21].

The $K \bar{K}$ decay mode of $f_{0}(1500)$ was observed meanwhile $[4,8]$ and is somewhat stronger than the upper limit from bubble chamber experiments, but still much weaker than the $\pi \pi$ mode. This indicates that $f_{0}(1500)$, if interpreted as a $q \bar{q}$ state, can have only a small $s \bar{s}$ component. Also, the missing $s \bar{s}$ scalar has now been observed: the longstanding controversy on the spin of the $f_{J}(1710)(J=0$ or 2$)$ was lifted in favour of $J=0$ [8,22]. The early amplitude analysis of former central production data assumed the spin $2 f_{2}^{\prime}(1525)$ around $1500 \mathrm{MeV}$, but no $f_{0}(1500)$. An updated analysis along the lines of ref. [16], but using instead of Crystal Barrel data more recent results from central collisions, leads to $83 \% s \bar{s}$ in $f_{0}(1710)$ and $60 \% u \bar{u}+d \bar{d}$ in $f_{0}(1370)$, while $f_{0}(1500)$ contains the largest fraction of glue (48 $\%)[23]$.

The purpose of this letter is to discuss the impact of these and other new data on the quark structures of $f_{0}(1500)$ and $f_{0}(1710)$. In particular, we will show that for $f_{0}(1500)$ the $K \bar{K}$ and $\gamma \gamma$ data do not appear to be consistent with a dominantly quarkonium state, while the $K \bar{K}$ data suggest a large $s \bar{s}$ component in the $f_{0}(1710)$ wavefunction.

\section{Couplings to two pseudoscalar mesons}

The branching ratios for $f_{0}(1500)$ decay into $\pi \pi, K \bar{K}$ and $\eta \eta$ have been measured by several groups: Crystal Barrel quotes from a coupled channel analysis of the $3 \pi^{0}, \pi^{0} \pi^{0} \eta$ and $\eta \eta \pi^{0}$ data in $\bar{p} p$ annihilation at rest [24] and from the $\pi^{0} K_{L} K_{L}$ channel [4]: 


$$
\begin{aligned}
\Gamma(\eta \eta) / \Gamma(\pi \pi) & =0.157 \pm 0.062, \\
\Gamma(K \bar{K}) / \Gamma(\pi \pi) & =0.119 \pm 0.032
\end{aligned}
$$

while WA102 reports in $p p$ central collisions [10]

$$
\begin{aligned}
\Gamma(\eta \eta) / \Gamma(\pi \pi) & =0.18 \pm 0.03 \\
\Gamma(K \bar{K}) / \Gamma(\pi \pi) & =0.33 \pm 0.07 .
\end{aligned}
$$

The $\eta \eta / \pi \pi$ ratio was also measured by Crystal Barrel with $900 \mathrm{MeV} / \mathrm{c}$ antiprotons [25]: $0.080 \pm 0.033$. The preliminary results for $K \bar{K} / \pi \pi$ from the Obelix collaboration is $0.24 \pm 0.02$ [26]. For $K \bar{K} / \pi \pi$ the agreement between experiments is not particularly good. However, branching ratios are sensitive to interference effects and are not defined unambiguously. The apparent discrepancy should therefore not be overemphasized. The disagreement is perhaps due to the nearby $a_{0}(1450)$ which absorbs a significant fraction of the $K \bar{K}$ rate in Crystal Barrel data, but is not required by the WA102 data, and reported at the lower mass of $1300 \mathrm{MeV}$ for the Obelix data. In any case, all data indicate that the coupling of $f_{0}(1500)$ to $K \bar{K}$ is small compared to $\pi \pi$.

To quantify this result we recall the formulae to compute the partial width of a scalar (or tensor) meson decaying into two pseudoscalar mesons $M_{1}$ and $M_{2}$ [16]

$$
\Gamma\left(M_{1} M_{2}\right)=\gamma^{2}\left(M_{1} M_{2}\right) \times q^{2 \ell+1} \times \exp \left(-q^{2} / 8 \beta^{2}\right),
$$

with $\beta \simeq 0.5 \mathrm{GeV} / \mathrm{c}$, where $q$ is the breakup momentum and $\ell$ the relative angular momentum (0 or 2). The couplings $\gamma^{2}$ are derived from SU(3) and read, up to a common multiplicative constant:

$$
\begin{aligned}
\gamma^{2}(\pi \pi) & =3[\cos \alpha]^{2}, \\
\gamma^{2}(K \bar{K}) & =[\cos \alpha(1-\sqrt{2} \tan \alpha)]^{2}, \\
\gamma^{2}(\eta \eta) & =\left[\cos \alpha\left(\cos ^{2} \phi-\sqrt{2} \tan \alpha \sin ^{2} \phi\right)\right]^{2} .
\end{aligned}
$$

Here $\alpha=54.7^{\circ}+\theta$ and $\phi=54.7^{\circ}+\theta_{P S}$. The angle $\theta$ is the octet-singlet mixing angle in the nonet of the decaying meson and $\theta_{P S}$ the mixing angle in the pseudoscalar nonet. We shall use the value $\theta_{P S}=-(17.3 \pm 1.8)^{\circ}$ measured from $\bar{p} p$ annihilation rates into two pseudoscalar mesons [27].

The wavefunction of the isoscalar $q \bar{q}$ meson, say $f^{\prime}$, ist then given by

$$
\left|f^{\prime}\right\rangle=\cos \alpha|n \bar{n}\rangle-\sin \alpha|s \bar{s}\rangle \quad \text { with } \quad|n \bar{n}\rangle \equiv \frac{u \bar{u}+d \bar{d}}{\sqrt{2}}
$$




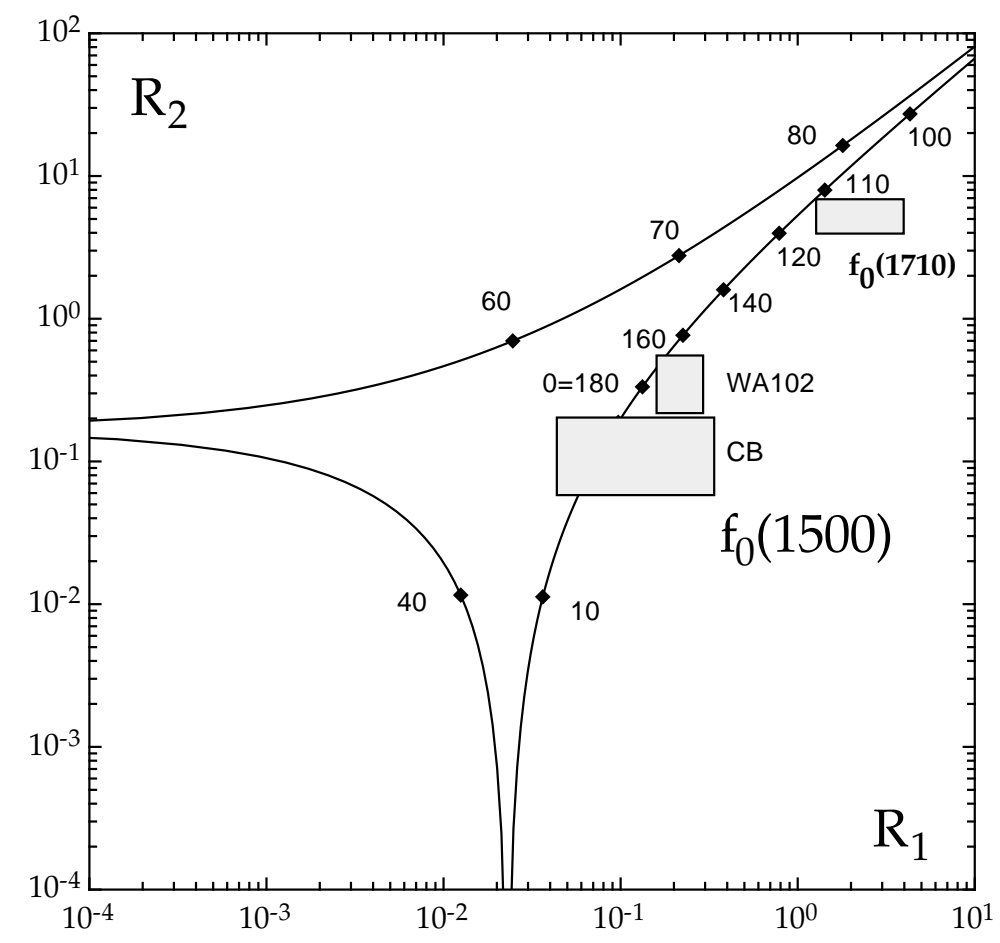

Fig. 1. Predicted relative coupling $R_{2}=\gamma^{2}(K \bar{K}) / \gamma^{2}(\pi \pi)$ vs. $R_{1}=\gamma^{2}(\eta \eta) / \gamma^{2}(\pi \pi)$ (curve) compared to data ( $2 \sigma$ boundaries) from Crystal Barrel (CB) and WA102 for the $f_{0}(1500)$ and from WA102 for the $f_{0}(1710)$. The numbers on the curve indicate values of the mixing angle $\alpha$ in degrees.

Hence for $\alpha=0$ the meson is pure $n \bar{n}$ and for $\alpha=90^{\circ}$ pure $s \bar{s}$ (ideal mixing). These prescriptions provide a very good description e.g. of the measured tensor meson decays [16], leading to a tensor mixing angle $\theta \simeq 27^{\circ}$, in accord with the linear and quadratic mass formulae. Note that, as shown in ref. [16], possible SU(3) breaking effects are small, and are accordingly neglected in our formulae (6) (hence the parameter $\rho$ in the appendix A of ref. [16] is set to unity).

Let us calculate the measured ratios $R_{1} \equiv \gamma^{2}(\eta \eta) / \gamma^{2}(\pi \pi)$ and $R_{2} \equiv \gamma^{2}(K \bar{K}) / \gamma^{2}(\pi \pi)$ using eqn. (5). We obtain

$$
R_{1}=0.196 \pm 0.077 \text { and } 0.227 \pm 0.035
$$

for Crystal Barrel and WA102, respectively, while

$$
R_{2}=0.139 \pm 0.037 \text { and } 0.375 \pm 0.082
$$

Note that for a pure $u \bar{u}+d \bar{d}$ state $R_{2}$ is expected to be $1 / 3$. Figure 1 shows the predicted dependence of the ratios $R_{1}$ and $R_{2}$ on the angle $\alpha$ for isoscalar mesons and the experimental results for $f_{0}(1500)$. We find that $\alpha \simeq 0$ (or $180^{\circ}$ ). The $\eta \eta^{\prime}$ decay rate from Crystal Barrel is also consistent with $\alpha \simeq 0$ [28]. If interpreted as a $q \bar{q}$ state the meson $f_{0}(1500)$ is therefore clearly dominantly $n \bar{n}$. 
Two comments are in order here. First, this conclusion is not sensitive to a reasonable departure ( $\sim 20 \%$ ) of the SU(3) breaking $\rho$ from unity. Second, assuming instead a pseudoscalar mixing angle $\theta_{P S}=-10^{\circ}$, as suggested by the quadratic mass formula for the $0^{-+}$nonet [1], leads to a very poor agreement of the measured $R_{1}$ and $R_{2}$ with SU(3) for any angle $\alpha$. In this case, it is difficult to reconcile $f_{0}(1500)$ with a $q \bar{q}$ state.

So far, only one experiment, WA102, measured the corresponding ratios of partial widths for the $f_{0}(1710)$ meson and reported [10]

$$
\begin{aligned}
\Gamma(\eta \eta) / \Gamma(\pi \pi) & =2.4 \pm 0.6 \\
\Gamma(K \bar{K}) / \Gamma(\pi \pi) & =5.0 \pm 0.7
\end{aligned}
$$

leading to the ratios $R_{1}=2.63 \pm 0.66$ and $R_{2}=5.37 \pm 0.75$, which are also shown in fig. 1 . The data are consistent with a state dominantly $s \bar{s}(\sim 86 \%$ for $\alpha=112^{\circ}$ ). Furthermore, a pure (ideally mixed) $s \bar{s}$ meson should not be produced in $\bar{p} p$ annihilation due to the OZI rule and, indeed, Crystal Barrel does not observe $f_{0}(1710)$ in $\bar{p} p$ annihilation at $900 \mathrm{MeV} / \mathrm{c}$, while $f_{0}(1500)$ is produced copiously [25].

\section{Couplings to two photons}

The L3 and ALEPH collaborations at LEP have reported new results in $\gamma \gamma$ collisions around the $Z^{0}$ pole and at higher energies $[14,15]$. The $f_{2}(1270)$ is seen by ALEPH in the $\gamma \gamma \rightarrow \pi^{+} \pi^{-}$mass spectrum, but neither $f_{0}(1500)$ nor $f_{0}(1710)$ are observed and $95 \%$ confidence level upper limits of $310 \mathrm{eV}$, respectively 550 $\mathrm{eV}$ are given for the formation and decay of these states into $\pi^{+} \pi^{-}$. According to Crystal Barrel, $4 \pi$ decays account for more than half of the decay rate of $f_{0}(1500)$. The $\pi^{+} \pi^{-}$decay branching ratio was measured: a compilation from early data [28] gives $0.193 \pm 0.050$, in agreement with a more recent $0.226 \pm 0.079$ from an extensive analysis of $\bar{p} p$ annihilation into $5 \pi$ [7]. Using the latter and combining with ALEPH we therefore obtain for the upper limit

$$
\Gamma\left(f_{0}(1500) \rightarrow \gamma \gamma\right) \leq 1.4 \mathrm{keV}
$$

at the $95 \%$ confidence level.

The L3 collaboration measuring $\gamma \gamma \rightarrow K_{s} K_{s}$ observes the $f_{2}(1270)$ and the $a_{2}(1320)$, the $f_{2}^{\prime}(1525)$, and a dominantly spin 2 signal around $1750 \mathrm{MeV}$, which is assigned to the $f_{J}(1710)$ (with, however, $J=2$ ). The measured partial width is

$$
\Gamma\left(f_{2}(1710) \rightarrow \gamma \gamma\right) \times \Gamma\left(f_{2}(1710) \rightarrow K \bar{K}\right) / \Gamma_{\text {tot }}=49 \pm 17 \mathrm{eV}
$$


However, roughly $25 \%$ of the signal is due to $J=0$. Assuming this signal to come from the $f_{0}(1710)$ one obtains [29]

$$
\Gamma\left(f_{0}(1710) \rightarrow \gamma \gamma\right) \times \Gamma\left(f_{2}(1710) \rightarrow K \bar{K}\right) / \Gamma_{\text {tot }}=130 \pm 96 \mathrm{eV}
$$

A spin 0 signal at $1500 \mathrm{MeV}$ is totally excluded by the L3 data, but the corresponding, presumably very small, upper limit for $f_{0}(1500)$ is not given. We shall argue below that the spin 2 component could instead be due to the (isovector) radial excitation $a_{2}(1700)$ and that the spin 0 component is consistent with a mainly $s \bar{s}$ $f_{0}(1710)$.

The two-photon width of the isoscalar meson $f^{\prime}$ (eqn. (7)) with mass $m$ is given within $\mathrm{SU}(3)$ by

$$
\Gamma_{\gamma \gamma}=c(5 \cos \alpha-\sqrt{2} \sin \alpha)^{2} m^{3}
$$

which vanishes for $\alpha=74^{\circ}$, and where $c$ is a nonet constant (for the isoscalar partner $f$ in the nonet replace $\alpha$ by $\alpha+90^{\circ}$ ). This formula is quite reliable as it reproduces the measured two-photon widths of the $2^{++}$mesons $f_{2}(1270)$ and $f_{2}^{\prime}(1525)$ and those of the $0^{-+}$isoscalars $\eta$ and $\eta^{\prime}$ (the latter albeit with $10 \%$ SU(3) violating corrections). To gain confidence in the formula, let us examine the predicted ratio $\Gamma_{\gamma \gamma}\left(f_{2}^{\prime}\right) / \Gamma_{\gamma \gamma}\left(f_{2}\right)$ as a function of $\alpha$. This is shown in fig. 2 and compared to the measured ratio of $0.0328 \pm 0.0062[1,14]$. This ratio is extremely sensitive to the tensor mixing angle and one obtains the accurate value $\theta=(27.3 \pm 0.8)^{\circ}$, in excellent agreement with the mass formulae.

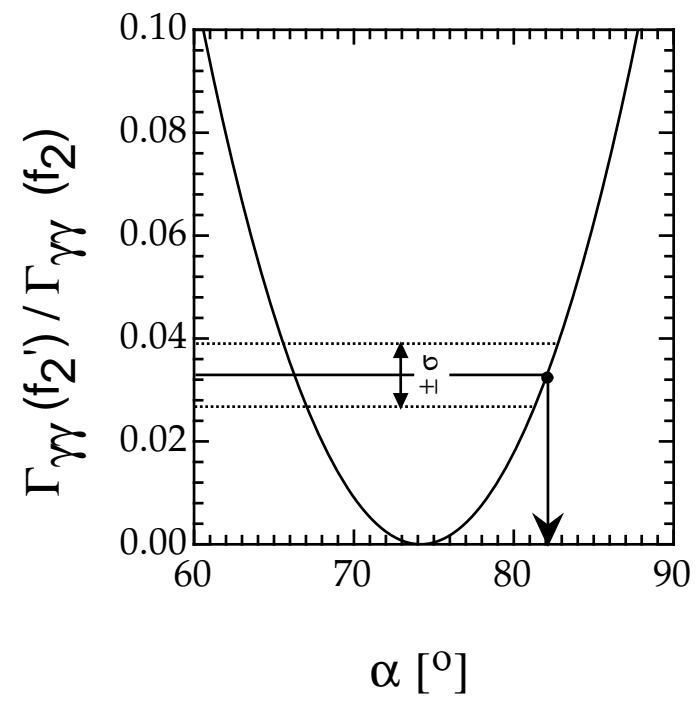

Fig. 2. Predicted ratio of two-photon widths for the $f_{2}(1270)$ and $f_{2}^{\prime}(1525)$ mesons as a function of $\alpha$. Note the twofold ambiguity. The measured value of $\alpha$ consistent with the mass formulae is shown by the arrow. 
Also, we find from eqn. (15) and the two-photon partial width $\Gamma_{\gamma \gamma}=9 \mathrm{c} \mathrm{m}^{3}$ for the isovector meson $a$ the well-known ratios of partial widths which read apart from $m^{3}$ factors

$$
\Gamma_{\gamma \gamma}(a): \Gamma_{\gamma \gamma}(f): \Gamma_{\gamma \gamma}\left(f^{\prime}\right)=9: 25: 2
$$

and which hold in the case of ideal mixing. Thus, as will become crucial in the following discussion, a pure $s \bar{s}$ isoscalar has a two-photon width an order of magnitude smaller than its $n \bar{n}$ partner.

The constant $c$ for the tensor nonet can be calculated from e.g. the two-photon width of $f_{2}^{\prime}(1525)$ [14]: $c=4.9 \times 10^{-14} \mathrm{MeV}^{-2}$ which, in turn, leads to a predicted two-photon partial width of $1.0 \mathrm{keV}$ for the $a_{2}(1320)$, in excellent agreement with the measured value [1].

Let us now deal with scalar mesons. In a non-relativistic calculation the two-photon width is given by [30]

$$
\Gamma_{\gamma \gamma}\left(0^{++}\right)=k\left(\frac{m_{0}}{m_{2}}\right)^{3} \Gamma_{\gamma \gamma}\left(2^{++}\right)
$$

with obvious notations. Here the factor $k=15 / 4$ stands for spin multiplicities. How reliable is this relation? It can be checked with recent data on the charmonium states $\chi_{c 2}$ and $\chi_{c 0}$ : the ratio of $\chi_{c 0}$ to $\chi_{c 2}$ two-photon widths is measured to be $6.7 \pm 2.1$ while eqn. (17) predicts the somewhat smaller value of 3.3. Relativistic calculations lead to a smaller value of scalar two-photon widths with $k \simeq 2$ [31]. Assuming that $f_{0}(1370)$ is the mainly $n \bar{n}$ scalar, one then predicts with formula (17) a two-photon width of $5.7 \mathrm{keV}$, in good agreement with analyses of $\gamma \gamma \rightarrow \pi \pi$ [32].

We shall therefore assume that for $k$ in the range $2 \leq k \leq 15 / 4$ formula (17) provides a reliable estimate of scalar two-photon widths. Figure 3 then shows the two-photon width expected for a $0^{++}$isoscalar at $1500 \mathrm{MeV}$ as a function of $\alpha$, calculated from eqns. (15) and (17), together with the 95\% confidence level upper limit (12) from ALEPH. Assuming a $q \bar{q}$ structure, the lack of $f_{0}(1500)$ production in $\gamma \gamma$ implies that this state must be mainly $s \bar{s}\left(50^{\circ} \leq \alpha \leq 100^{\circ}\right)$. This is, however, in clear contradiction with the $n \bar{n}$ dominance discussed in the previous section and therefore suggests that $f_{0}(1500)$ is of a different nature.

Little can be said about $f_{0}(1710)$ from the ALEPH data, since its decay branching ratio into $\pi^{+} \pi^{-}$is not known. Assuming $\alpha \sim 112^{\circ}$ from the previous section, we find from fig. 3 and the ALEPH two-photon upper limit of $550 \mathrm{eV}$ a $\pi^{+} \pi^{-}$ branching ratio of at most 5 to $10 \%$, hence small, as expected for a mainly $s \bar{s}$ state. 


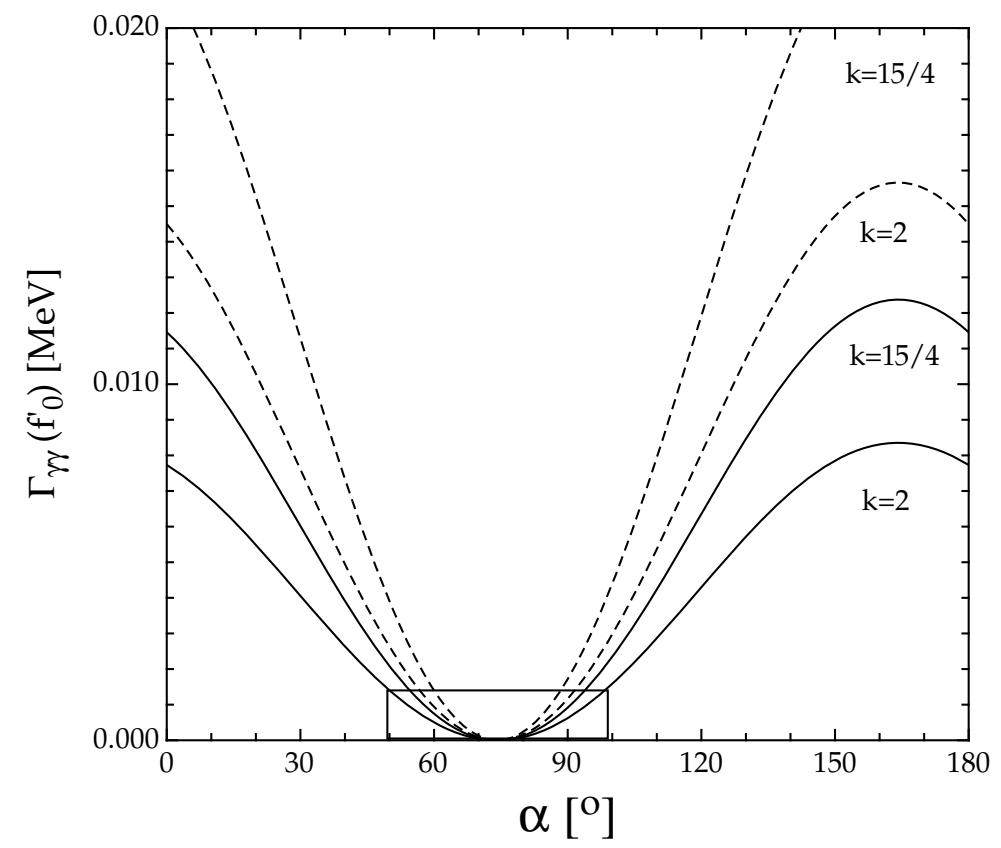

Fig. 3. Predicted two-photon width for the $f_{0}(1500)$ (full curves) and the $f_{0}(1710)$ (dashed curves) assuming a $q \bar{q}$ structure. The box shows the $95 \%$ confidence level upper limit from ALEPH for the $f_{0}(1500)$.

Let us now turn to the L3 data [14] and to the signal observed around $1750 \mathrm{MeV}$. Since decay branching ratios into $K \bar{K}$ are not known, only qualitative statements can be made. Assuming that the spin two component is due to the $a_{2}(1700)$ reported by L3 in $\gamma \gamma \rightarrow 3 \pi$ [33] and by Crystal Barrel in $\bar{p} p \rightarrow \pi^{0} \pi^{0} \eta$ [25] one would obtain from the partial width (13) and with, say, a 5\% decay branching ratio into $K \bar{K}$, a two-photon width of $\simeq 1 \mathrm{keV}$. These numbers are comparable to those for the ground state $a_{2}(1320)$. As far as the spin zero signal is concerned, the upper limit (14) can be accommodated by a nearly pure $s \bar{s}$ with a large $K \bar{K}$ decay branching ratio (see fig. 3 ).

\section{Discussion and conclusions}

As we have argued, the measured $\pi \pi, \eta \eta, \eta \eta^{\prime}$ and $K \bar{K}$ decay rates of $f_{0}(1500)$ in $\bar{p} p$ annihilation and central production are consistent with those expected for a $u \bar{u}+d \bar{d}$ state. On the other hand, $f_{0}(1500)$ is not produced in $\gamma \gamma$ collisions, with an upper limit at the level expected for a mainly $s \bar{s}$ state. This contradiction suggests that $f_{0}(1500)$ is not a $q \bar{q}$ state. A four-quark state is unlikely since its two-photon width is not significantly smaller than for a $q \bar{q}$ state. For example, a partial width of $\simeq 0.6 \mathrm{keV}$ is predicted in ref. [34] for the prominent candidate $a_{0}(980)$, while formula (17) gives $0.8 \mathrm{keV}$ for a $q \bar{q}$ state (using $1 \mathrm{keV}$ for the $a_{2}(1320)$ and $k=2$ ).

The natural explanation is that $f_{0}(1500)$ is a gluonium state, since the production 
of glue in $\gamma \gamma$ collisions is suppressed. Its mass also lies in the correct range, lattice gauge calculations predicting the ground state scalar glueball to be at $1611 \pm 30 \pm$ $160 \mathrm{MeV}$ [17].

The $f_{0}(1710)$ appears to be dominantly $s \bar{s}$ from $\pi \pi, \eta \eta$ and $K \bar{K}$ data in central collisions. This is consistent with its absence in $\bar{p} p$ annihilation where scalars are copiously produced and no mechanism is known preventing the production of $u \bar{u}+$ $d \bar{d}$ scalars. We note that, surprisingly for an $s \bar{s}$ state, no signal for $f_{0}(1710)$ was reported earlier in $K^{-} p \rightarrow K_{S} K_{S} \Lambda$ interactions [35]. However, the assumption in the analysis was that its spin was two. The $s \bar{s}$ dominance is consistent with (but not required by) $\gamma \gamma$ data, since only few decay branching ratios have been measured for this state. In particular, the absolute two-body decay rates are not known and are urgently needed from future experiments.

If one now assumes $a_{0}(1450), f_{0}(1370), f_{0}(1710)$ and $K_{0}^{*}(1430)$, one obtains from the linear mass formula

$$
\tan ^{2} \theta=\frac{4 m\left(K_{0}^{*}\right)-m\left(a_{0}\right)-3 m\left[f_{0}(1710)\right]}{3 m\left[f_{0}(1370)\right]+m\left(a_{0}\right)-4 m\left(K_{0}^{*}\right)}
$$

the $0^{++}$mixing angle $\alpha=\theta+54.7^{\circ} \simeq 114^{\circ}$ for a low mass $(1300 \mathrm{MeV}) a_{0}$ or $\simeq 127^{\circ}$ for a high mass $(1470 \mathrm{MeV}) a_{0}[1]$. We have assumed for $f_{0}(1370)$ a mass of $1360 \mathrm{MeV}$ [28]. The mixing angle is also sensitive to the mass of this state which is not well known [1]. It is interesting to note that these values of $\alpha$ are in the correct range, see the data on the $f_{0}(1710)$ in fig. 1 above.

The glueball is the $f_{0}(1500)$ in our model. This may be too simple minded, since the two $q \bar{q}$ isoscalars are likely to mix with the nearby glueball. Nonetheless these results strongly favour the scenario where $f_{0}(1500)$ is largely glue and $f_{0}(1710)$ dominantly $s \bar{s}[16,23]$, and disfavour $f_{0}(1710)$ being the glueball [21]. More quantitative statements will have to await more accurate $\gamma \gamma$ data and theoretical guidance on the production rate of glueballs in electromagnetic processes.

\section{References}

[1] Review of Particle Physics: D. Groom et al., Eur. Phys. J. 15 (2000) 1;

K. Hagiwara et al., 2002 issue (in preparation)

[2] C. Amsler et al., Phys. Lett. B 342 (1995) 433

[3] A. Bertin et al., Phys. Lett. B 408 (1997) 476

[4] A. Abele et al., Phys. Lett. B 385 (1996) 425

[5] C. Amsler et al., Phys. Lett. B 353 (1995) 571 
[6] C. Amsler et al., Phys. Lett. B 340 (1994) 259

[7] A. Abele et al., Eur. Phys. J. C 21 (2001) 261

[8] D. Barberis et al., Phys. Lett. B 462 (1999) 462

[9] D. Barberis et al., Phys. Lett. B 471 (2000) 429

[10] D. Barberis et al., Phys. Lett. B 479 (2000) 59

[11] D. Barberis et al., Phys. Lett. B 471 (2000) 440

[12] P. L. Frabetti et al., Phys. Lett. B 407 (1997) 79

[13] D. V. Bugg et al., Phys. Lett. B 353 (1995) 378

[14] M. Acciarri et al., Phys. Lett. B 501 (2001) 173

[15] R. Barate et al., Phys. Lett. B 472 (2000) 189

[16] C. Amsler and F. E. Close, Phys. Rev. D 53 (1996) 295

[17] C. Michael, Hadron 97 Conf., AIP Conf. Proc. 432 (1998) 657

[18] R. L. Jaffe, Phys. Rev. D 15 (1977) 267, 281; J. Weinstein, N. Isgur, Phys. Rev. D 41 (1990) 2236; N. N. Achasov and V. V. Gubin, Phys. Rev. D 56 (1997) 4084

[19] J. A. Oller et al., Phys. Rev. D 59 (1999) 074001

[20] N.A. Törnquist, Z. Phys. C68 (1995) 647; M. Boglione and M. R. Pennington, Phys. Rev. Lett. 79 (1997) 1998; A.V. Anisovich, V. V. Anisovich, A. V. Sarantsev, Z. Phys. A357 (1997) 123; P. Minkowski and W. Ochs, Eur. Phys. J. C9 (1999) 283

[21] D. Weingarten, Nucl. Phys. Proc. Suppl. 53 (1997) 232; 63 (1998) 194; 73 (1999) 249

[22] W. Dunwoodie, Hadron 97 Conf, AIP Conf. Proc. 432

[23] F. E. Close, A. Kirk, Phys. Lett. B 483 (2000) 345

[24] C. Amsler et al., Phys. Lett. B 355 (1995) 425

[25] C. Amsler et al., Eur. Phys. J. C 23 (2002) 29

[26] S. Vecchi, private communication (to be published)

[27] C. Amsler et al., Phys. Lett. B 294 (1992) 451

[28] C. Amsler, Rev. Mod. Phys. 70 (1998) 1293

[29] S. Braccini, PhD thesis No. 3255, University of Geneva, 2001

[30] K. A. Tumanov, Zh. Eksp. Teor. Fiz. 25 (1953) 385;

A. I. Alekseev, Zh. Eksp. Teor. Fiz. 34 (1958) 1195

[31] Z. P. Li, F. E. Close and T. Barnes, Phys. Rev. D 43 (1991) 2161;

L. Bergström, G. Hulth, and H. Snellman, Z. Phys. C 16 (1983) 263 
[32] M. Boglione and M. R. Pennington, Eur. Phys. J. C9 (1999) 11;

D. Morgan and M. R. Pennington, Z. Phys. C 48 (1990) 623

[33] M. Acciarri et al., Phys. Lett. B 413 (1997) 147

[34] T. Barnes, Phys. Lett. 165 B (1985) 434

[35] D. Aston et al., Nucl. Phys. B 301 (1988) 525 